\title{
Radiographic Assessment of Distal Radius Fracture Treatment Outcomes Following Various Types of Fracture Stabilization in Elderly Patients
}

\section{Jarosław Olech}

Provincial specialist hospital in Legnica

\section{Bartosz Kopczyński}

Opole University: Uniwersytet Opolski

Piotr Morasiewicz ( $\square$ morasp@poczta.onet.pl)

Opole University: Uniwersytet Opolski https://orcid.org/0000-0002-7587-666X

\section{Research Article}

Keywords: distal radius, fracture, radiographic, volar plate, Kirschner wires, cast

Posted Date: July 9th, 2021

DOI: https://doi.org/10.21203/rs.3.rs-695727/v1

License: (c) (i) This work is licensed under a Creative Commons Attribution 4.0 International License.

Read Full License 


\section{Abstract}

Background: Distal radius fractures pose a serious problem due to their high incidence and can be treated with various methods. No specific distal radius fracture treatment is acknowledged to be the gold standard by orthopedic surgeons. The aim of study was to conduct a comprehensive radiographic assessment of treatment outcomes in patients with distal radius fractures following various types of stabilization in elderly patients.

Methods: We retrospectively assessed 122patients who underwent treatment for distal radial fracture in the years 2017and2018.There were three study groups: closed reduction with K-wire fixation (37patients),open reduction with volar plate fixation (42 patients),and closed reduction with cast immobilization (43patients). The mean age at the beginning of treatment was 73years. The mean followup period was 2 years and 7 months. The following radiological parameters were evaluated: union rate, time to union, time of fracture immobilization, fracture stabilization failure, and the development of adjacent-joint arthritis.

Results: There were no significant differences between the study groups in terms of union rate, time to union, and the development of adjacent-joint arthritis. After treatment, the lowest rates of posttraumatic intercarpal and carpometacarpal arthritis and the lowest rates of fracture stabilization failure in our study were observed in the plaster-cast group. Volar plate fixation was associated with the shortest duration of fracture stabilization. In comparison to the status from before treatment, all study groups showed increased rates of intercarpal and carpometacarpal arthritis after treatment.

Conclusions: Our radiographic assessments demonstrated similar outcomes, regardless of the stabilization method. In treating distal radius fractures, we achieved good radiographic treatment outcomes irrespective of the fracture stabilization method used.

\section{Background}

Distal radius fractures pose a serious problem due to their high incidence [1-12[. Depending on fracture morphology, distal fractures of the radius can be treated with various methods $[2,6,7,9,10,11,13-30]$. No specific distal radius fracture treatment is acknowledged to be the gold standard by orthopedic surgeons $[2,6,7,9,10,11,13,15,16,17,22,23,25-27]$. Basically immobilization in cast is indicated for simple fractures or less demanding patients as elderly patients. High-energy fractures with higher impact on adjacent structures in most cases require fixation as Volar plate or at least K wires. Some reports present better treatment outcomes in distal radius fracture treatment with the use of open reduction and volar plate fixation $[6,9,13,23,27]$ than with closed reduction and cast immobilization. Some reports addressing distal radius fracture treatment present better outcomes with the use of open reduction with volar plate fixation $[25,26]$ in comparison with closed reduction with K-wire fixation. In elderly patients Kilic prefers treatment via immobilization in a cast [24]. Some authors reported comparable treatment outcomes irrespective of the method used $[15,18]$. The use of volar plate fixation yielded better outcomes 
than other methods of fixation/immobilization during short-term follow-up; however, long-term follow-up showed outcomes for all fixation/immobilization methods to be comparable[11]. Moreover, elderly patients showed good clinical and functional outcomes with cast immobilization[11].

There have been no studies offering a comprehensive radiographic evaluation of distal radius fracture outcomes following the use of a volar plate, K-wires, and a plaster cast in elderly patients. Comparing results of various methods may be beneficial to discuss necessity of more frequent application of particular method. Obtained results may be correlated with functional results after individual treatment method. According to some authors, correct radiological parameters contribute to good clinical results[5, $26,29]$.

While assessing radiographic treatment outcomes, it is important to note any evidence of adjacent-joint arthritis[21,30]. It may help to conclude if invasive treatment with short immobilisation is better than conservative treatment and longer immibilisation or alternatively. The development of adjacent joint arthritis is associated with the limitation of their functions, reduced mobility and pain. This can lead to poorer treatment outcomes. Therefore, it is important to assess which method of stabilization of radial fractures causes the least development of adjacent joint arthritis after treatment. In our study, we wanted to check whether conservative treatment, which is safer, especially in the time of the COVID-19 pandemic and associated with lower costs in elderly patients with distal radius fractures, can give results similar to surgical treatment.

The aim of our study was to conduct a comprehensive radiographic assessment of treatment outcomes in elderly patients with distal radius fractures, with respect to stabilization via volar plating, K-wires, or a cast.

\section{Methods}

We retrospectively assessed 122 patients who underwent treatment for distal radial fracture in the years 2017 and 2018. Study inclusion criteria were: age over 60 years, treatment for a unstable, dislocated or multigramental distal radius fracture, availability of complete medical and radiographic records from the treatment period, and minimum posttreatment follow-up of 2 years. Study exclusion criteria were: under 60 years of age, bilateral distal radius fracture, upper limb injury in other locations besides the distal forearm, open fracture, metaphyseal and diaphyseal radial fracture, and crush fracture involving damage to soft tissues, vessels and verves. A total of 122 patients ( 91 women and 31 men) were included in the study. The mean age at the beginning of treatment was 73 years (ranging from 61 to 82 years). The mean follow-up period was 2 years and 7 months (ranging from 2 years 1 month to 3 years 4 months). The evaluated subgroups showed no significant differences in terms of patient age $(p=0.724)$. The study was approved by the local ethics committee. Informed consent was obtained from each patient.

The patients were divided into three groups based on the treatment method (open reduction with volar plate fixation, closed reduction with $\mathrm{K}$-wire fixation, and closed reduction with cast immobilization). All surgical procedures were performed by one of three orthopedic surgeons. The treatment method was 
chosen based on patient age, bone quality, extent of soft-tissue injury, fracture morphology, the shape of bone fragments, concomitant radial shortening and deformity.

In patients with good bone quality and good condition of the adjacent soft tissues, with marked radial shortening and deformity, unstable, or multigramental fracture the preferred method was open reduction with volar plate fixation. In patients with poor bone quality and poor condition of the adjacent soft tissues, in the case of marked radial shortening and deformity, unstable, or multigramental fracture the preferred method was closed reduction with K-wire fixation. In patients with marked radial shortening and deformity, unstable, or multigramental fracture, who did not agree to surgery or had contraindications for surgery, closed reduction with cast immobilization was performed.

The three study groups, formed based on the treatment method used were: Group 1 - closed reduction with K-wire fixation (37 patients), Group 2 - open reduction with volar plate fixation (42 patients), and Group 3 - closed reduction with cast immobilization (43 patients).

All patients who underwent surgical treatment received antibiotic prophylaxis, and surgeries were performed in a supine position, under general anesthesia, with tourniquet application $(250 \mathrm{~mm} \mathrm{Hg})$. Volar plate fixation of bone fragments was conducted via fluoroscopy-guided open reduction through a volar approach (Fig. 1). Group 1 patients underwent fluoroscopy-guided closed reduction, with subsequent percutaneous fixation with two K-wires introduced through the radial styloid process and one perpendicular wire inserted into the radius from the ulnar side (Fig. 2). Group 3 patients underwent closed reduction with immobilization of the upper limb in a below-elbow cast (Fig. 3).

The cast or K-wires were removed once adequate bone union was confirmed both radiographically and clinically. Once the cast or K-wires were removed, finger and wrist exercises were introduced, and the patients were advised to use the limb only to a limited extent for a period of 3-6 weeks. The level of physical activity for the limb was gradually increased, based on the radiographic and clinical evidence of bone remodeling progress at the fracture site.

Radiographic assessments were conducted at least 2 years after treatment completion and were based on medical records obtained during treatment and over the outpatient follow-up period. The following radiological parameters were evaluated: union rate, time to union, fracture stabilization failure, and the development of adjacent-joint arthritis.

Union rate was assessed based on radiographic and clinical criteria. The radiographic criterion of union was the presence of at least 3 out of 4 consolidated cortices or continuous trabecular bridging between the bone fragments in anteroposterior and lateral views of the wrist [7, 30]. Clinical criteria were the absence of the following: pain, pathological mobility, and antebrachial deformity on forcible attempts at movement at the fracture site. Nonunion was defined as failure to meet the above criteria by 6 months after fracture. 
Time to union was assessed based on anteroposterior and lateral wrist radiographs, with the use of the same clinical and radiographic criteria as those used for assessing union rate.

Stabilization failure was defined as bone fragment displacement after stabilization of the fracture $>10^{0}$ or/and leading to nonunion.

Adjacent-joint arthritis was assessed with Knirk and Jupiter's criteria, based on anteroposterior and lateral wrist radiographs taken at three time points: at baseline, at the time of the fracture, and at the final followup visit $[14,29]$. The assessed joints were intercarpal (scaphotrapezial-trapezoid and scapholunate) and carpometacarpal (capitometacarpal and hamatometacarpal). The arthritis status from before treatment was compared with that observed after treatment. These radiographic findings were used as a means of comparison between the individual study groups.

The non-parametric Kruskal-Wallis test was used to assess the distribution of numerical variables and test the equality of median values in the analyzed groups.

The chi-square test was used to assess the distribution of categorical variables in the individual study groups and determine the statistical significance of any differences. The level of significance was adopted at $\mathrm{a}=0.05$, with the levels of significance $0.05<p<0.1$ interpreted as a trend towards statistical significance. The calculations were conducted with Statistica 10.0 software.

\section{Results}

According to the $\mathrm{AO}$ foundation classification, in the group with cast immobilization group $11,6 \%$ of patients had type A fractures, $44,2 \%$ of patients had type B fractures, and $44,2 \%$ type $C$, in the group with plate stabilization, $9,5 \%$ of patients had type $A$ fractures, $42,9 \%$ of patients had type $B$ fractures and 47,6 $\%$ type $C$ fractures, while in the group with $K$-wire stabilization, $10,8 \%$ of patients had type $A$ fractures, $43,2 \%$ of patients had type $B$ fractures and $46 \%$ type $C$ fractures.

Bone union was achieved in all patients whose fractures were stabilized percutaneously via K-wires. The highest rate of nonunion (4.5\%) was observed in the volar plate group, and the rate of nonunion in the cast group was (3.1\%); (Table 1). Nonetheless, these differences were not statistically significant (Table 1). 
Table 1

Detailed results of the radiological assessment of individual subgroups

\begin{tabular}{|c|c|c|c|c|}
\hline analyzed variable (Mean) & $\begin{array}{l}\text { Kirschner } \\
\text { group }\end{array}$ & $\begin{array}{l}\text { Plate } \\
\text { group }\end{array}$ & $\begin{array}{l}\text { Cast } \\
\text { group }\end{array}$ & $\begin{array}{l}p \\
\text { value }\end{array}$ \\
\hline Total union [weeks] & 8,666 & 8,5 & 8,376 & 0,556 \\
\hline Immobilization [weeks] & 6,333 & 5,136 & 5,83 & $0,017 *$ \\
\hline Age of patients [years] & 71,221 & 74,343 & 73,38 & 0,724 \\
\hline Achieving union [\%] & 100 & 95,455 & 96,923 & 0,761 \\
\hline Intercarpal arthrosis before treatment [\%] & 25 & 40,909 & 36,923 & 0,644 \\
\hline Intercarpal arthrosis after treatment [\%] & 41,667 & 59,091 & 50,769 & 0,608 \\
\hline $\begin{array}{l}\text { Carpometacarpal arthrosis before treatment } \\
\text { [\%] }\end{array}$ & 41,667 & 63,636 & 64,615 & 0,288 \\
\hline $\begin{array}{l}\text { Carpometacarpal arthrosis after treatment } \\
\text { [\%] }\end{array}$ & 75 & 81,818 & 73,846 & 0,726 \\
\hline \multirow[t]{2}{*}{ Destabilization [\%] } & 33,333 & 27,273 & 0,769 & $0,000 *$ \\
\hline & $*-p<0,05$ & & & \\
\hline
\end{tabular}

The mean duration of fracture immobilization for volar plate fixation, cast immobilization, and closed reduction with K-wire stabilization was $5.13,5.83$, and 6.33 weeks, respectively; (Table 1 ). The duration of treatment was significantly shorter in the volar plate group $(p=0.017)$; (Table 1$)$.

The mean time to complete union in the volar plate, cast, and K-wire groups was 8.5, 8.37, and 8.66 weeks, respectively;(Table 1). These values showed no significant differences (Table 1).

Our assessment for any evidence of fracture stabilization failure revealed that none of the patients treated with a plaster cast exhibited bone fragment displacement, whereas $27 \%$ of patients from the volar plate group showed screw migration, and $33 \%$ of patients treated with $\mathrm{K}$-wires exhibited K-wire migration (Table 1). The plaster-cast group showed the lowest proportion of fracture stabilization failure, which was statistically significant $(p=0) ;($ Table 1$)$.

Before treatment, intercarpal arthritis was observed in $25 \%$ of K-wire patients, in $41 \%$ of volar plate patients, and in $37 \%$ of plaster cast patients(Table 1). There were no statistically significant differences (Table 1). The rates of intercarpal arthritis, have significantly increased in all evaluated groups after treatment, with $421 \%, 59 \%$, and $51 \%$ of patients showing posttreatment intercarpal arthritis in the K-wire, volar plate, and plaster-cast group, respectively (Table 1). Statistically the inter-group differences were not significant (Table 1). $37 \%$ of the total number of patients exhibited pre-treatment intercarpal arthritis. A 
noticeable increase after treatment was found up to $51 \%$ of all patients; this difference was statistically significant $(p=0.027)$.

Prior to treatment, carpometacarpal arthritis was present in $42 \%$ of K-wire patients, in $64 \%$ of volar plate patients, and in $65 \%$ of plaster-cast patients (Table 1 ). The differences were not statistically significant (Table 1). After treatment, the rates of carpometacarpal arthritis were shown to be higher in all study groups, with $75 \%, 82 \%$, and $74 \%$ of patients showing posttreatment carpometacarpal arthritis in the Kwire, volar plate, and plaster-cast group, respectively (Table 1). The inter-group differences in terms of the rates of posttreatment carpometacarpal arthritis were not statistically significant (Table 1). In general, $63 \%$ of the total number of patients exhibited pre-treatment carpometacarpal arthritis. After treatment, carpometacarpal arthritis was detected in $75 \%$ of all patients. This increase appears to be considerable, despite the $p$-value calculated based on the available data being greater than $0.05(p=0.095)$.

\section{Discussion}

With the estimated proportion of distal radius fractures ranging from $15-21 \%$ of all fractures, the distal radius is the most common fracture location [1-12]. There is no consensus as to the recommended treatment of distal radius fractures $[2,6,7,9,11,13,15-18,22,23,25-27]$.

The use radiographic parameters in evaluating treatment outcomes is important to both doctors and patients $[7,8,9,14,15,17,19,24,27]$. The aim of our work was a comprehensive radiographic assessment of various types of stabilization in elderly patients with distal radius fractures and obtaining information on which method gives the best radiological results.

Fan et al. reported bone union in all 12 patients treated with volar plate fixation [17]. Kilic reported bone union in all evaluated patients treated via immobilization with a plaster cast [24]. Yin et al. reported achieved union in all patients with distal radius fracture treated with a plaster cast[28]. In our study the best union rates were observed in the group of patients treated via percutaneous K-wire stabilization, all of whom achieved bone union, whereas the highest proportion of nonunion (4.5\%) was observed in the group treated via open reduction with volar plate fixation, which was associated with fracture stabilization failure. We would like to mention that the lack of bone union among the patients treated with a cast was observed in elderly patients and those who exhibited considerable radial deformity or whose fracture had not been appropriately corrected.Statistical analysis showed no significant differences between the study groups in terms of union rates.

Fan et al. reported bone union achieved in a group of 12 patients after a mean follow-up of 3 months [17]. Kilic reported union after 3-5 weeks in patients treated via immobilization with a plaster cast [24]. Our study groups did not differ significantly in terms of the mean time to complete union depending on the method of stabilization. Our time to complete union data were similar to the data in the relevant literature $[17,24]$. 
There is no consensus among orthopedic surgeons as to the required period of cast immobilization in distal radius fractures $[9,24,27]$. Various authors prefer an immobilization period of 4 weeks $[9,24], 5$ weeks [24], or 6 weeks $[9,27]$. In the relevant group of our study, the mean period of cast immobilization was 5.83 weeks, which is consistent with the data presented in literature $[9,24,27]$. The patients treated via volar plate fixation required fracture stabilization to be maintained for a significantly shorter period. Interestingly, this latter method ensures good statistical results with a shorter stabilization time. The group who required fracture stabilization for the longest period were the patients treated via closed reduction with K-wire stabilization.

In our study the lowest rate of fracture stabilization failure was noted in the group of patients immobilized with a cast, with not a single case observed. Conversely, the highest rate of fracture stabilization failure was observed in the group treated via percutaneous K-wire stabilization, with K-wire migration being the most common cause.

The only authors who assessed adjacent-joint arthritis after distal radius fracture were Lutz et al.; however, the study was limited to a single stabilization method [21]. Chung et al. reported posttreatment arthritis in $15.4 \%$ of patients who underwent volar plate fixation, in $14.3 \%$ of patients who underwent Kwire stabilization, in $17.7 \%$ of patients who underwent stabilization via an external fixator, and in $25 \%$ of patients treated with a plaster cast [6]. Katayama et al. observed a correlation between the development of osteoarthritis and such factors as abnormal radial inclination, abnormal volar tilt, and reduced wrist mobility [7]. In that study osteoarthritis was reported in $66.1 \%$ of patients following distal radius fracture fixation with a volar plate [7]. A study by Lameijer conducted in young patients at a mean age of 37 years showed posttraumatic arthritis in $37-50 \%$ of cases [8]. Lameijer et al. observed development of distal radioulnar joint arthritis in $32 \%$ of patients (at a mean age of 32 years) following distal radius fracture treatment [14]. Saving et al. observed distal radioulnar osteoarthritis in $42 \%$ of the distal radius fracture patients treated via volar plate fixation and in $28 \%$ of those treated via an external fixator [15]. Erhart et al. reported that a more pronounced posttreatment deformity at the distal radius correlated with higher stages of posttraumatic osteoarthritis at the distal radioulnar joint [19].

In our study the highest proportion of pretreatment intercarpal arthritis was observed in the volar plate fixation group ( $41 \%$ of patients), whereas the lowest proportion was observed in the K-wire group ( $25 \%$ of patients). Pretreatment arthritis was present in $37 \%$ of patients from the plaster-cast group. Following treatment, the proportion of patients with arthritis increased to a similar extent in all study groups (by 16 percentage points in the K-wire group, by 18 percentage points in the volar plate group, and by 14 percentage points in the plaster-cast group). In the case of carpometacarpal arthritis, there was a different pattern of progression from the pretreatment to posttreatment status. Prior to treatment, the lowest proportion of arthritis (41\%) was observed in the K-wire group, whereas arthritis rates in the plaster-cast and volar-plate groups were similar, with $64 \%$ of patients affected in either. The lowest proportion of posttreatment carpometacarpal arthritis was observed in the plaster-cast group (an increase by 10 percentage points). After treatment, the volar-plate group showed an 18-percentage point increase in arthritis, and the greatest increase (by 34 percentage points) in arthritis rates was observed in the K- 
wire group. After treatment of the fracture, all study groups showed increased rates of adjacent-joint arthritis.

One limitation of our study is its retrospective nature; however, this is a direct result of the subject matter being fractures, which are impossible to predict, thus the pretreatment assessment is very limited. The strengths of this study are a relatively large number of patients, the individual groups showing no differences in terms of patient age, all of the evaluated patients being treated by only three orthopedic surgeons, and the use of the same postoperative and rehabilitation protocols in the case of each patient. In order to eliminate interobserver variability, all radiographic measurements were conducted according to the same protocol by a single experienced specialist [14].In the future, we plan to prepare work on a larger number of patients and including functional assessment.

After treatment, the lowest rates of posttraumatic intercarpal and carpometacarpal arthritis and the lowest rates of fracture stabilization failure in our study were observed in the plaster-cast group. Volar plate fixation was associated with the shortest immobilisation through solid bone fixation. The study groups showed no significant differences in terms of union rates or time to complete union.

Our radiographic assesments demonstrated slightly better results in patients treated with immobilization in cast. This type of treatment is dedicated for fractures with lesser deformity or for less demanding patients (elderly). Displaced, multigramental fractures require better forms of reduction and stabilizations. This type of fractures are more difficult to achieve great radiolographic results. A group of difficult multigramental fractures and destabilization of metal implants have impacted final assesments and radiographic results of methods with internal and external fixation. Relatively good result in plaster cast group is most likely linked to absence of destabilisation of fixation.

In comparison to the status from before treatment, all study groups showed increased rates of intercarpal and carpometacarpal arthritis after treatment. Extended immobilisation is most likely responsible for increased rate of arthrosis in the plaster cast group and the K-wire group. Increased rate of arthrosis should be linked to high-energy complex type of fracture treated with Volar plate.

It is advisable to consider more frequent non-surgical treatment of the distal radius fractures in elderly patients, especially during the COVID-19 pandemic

\section{Conclusions}

Our detailed radiographic assessments demonstrated similar outcomes, regardless of the stabilization method. In treating distal radius fractures in elderly patients, we achieved good radiographic treatment outcomes irrespective of the fracture stabilization method used.

\section{Declarations}

Ethics approval and consent to participate: Not applicable. 
Consent for publication: Not applicable.

Availability of data and materials: The datasets used and/or analysed during the current study are available from the corresponding author on reasonable request.

Competing interests: The authors declare that they have no competing interests

Funding: No funding received for this study.

Authors' contributions: JO and PM carried out the concepts, design, definition of intellectualcontent, literature search, data acquisition, data analysis, and manuscript preparation. BK provided assistance for data acquisition, data analysis,and statistical analysis. All authors have performed manuscript review. All authors have read and approved the content of the manuscript.

Acknowledgements: Not applicable.

\section{References}

1. Kwon HY, Kim HH, Sung YK, Ha YC. Incidence and Mortality of Osteoporotic Fracture in Rheumatoid Arthritis in South Korea Using Nationwide Claims Data. J Bone Metab. 2019 May;26(2):97-104.

2. Ogunleye AA, Mullner DF, Skochdopole A, Armstrong M, Herrera FA. Remote Injuries and Outcomes After Distal Radius Fracture Management. Hand (N Y). 2019 Jan;14(1):102-106.

3. Ochi K, Go Y, Furuya T, Ikari K, Taniguchi A, Yamanaka H, Momohara S. Risk factors associated with the occurrence of distal radius fractures in Japanese patients with rheumatoid arthritis: a prospective observational cohort study. Clin Rheumatol. 2014 Apr;33(4):477-83.

4. Ali M, Eiriksdottir A, Murtadha M, Åkesson A, Atroshi I. Incidence of distal radius fracture in a general population in southern Sweden in 2016 compared with 2001. Osteoporos Int. 2020 Jan 13.

5. Talmaç MA, Görgel MA, Kanar M, Tok O, Özdemir HM. Comparison of three surgical methods in the treatment of intraarticular comminuted distal radius fractures: Volar locking plate, non-bridging external fixator, and bridging external fixator. Eklem Hastalik Cerrahisi. 2019 Dec;30(3):224-32.

6. Chung KC, Malay S, Shauver MJ, Kim HM; WRIST Group. Assessment of Distal Radius Fracture Complications Among Adults 60 Years or Older: A Secondary Analysis of the WRIST Randomized Clinical Trial. JAMA Netw Open. 2019 Jan 4;2(1):e187053.

7. Katayama T, Ono H, Omokawa S. Comparison of Five Years Clinical and Radiological Outcomes between Progressive and Non-Progressive Wrist Osteoarthritis after Volar Locking Plate Fixation of Distal Radius Fractures. J Hand Surg Asian Pac Vol. 2019 Mar;24(1):30-35.

8. Lameijer CM, Ten Duis HJ, Dusseldorp IV, Dijkstra PU, van der Sluis CK. Prevalence of posttraumatic arthritis and the association with outcome measures following distal radius fractures in nonosteoporotic patients: a systematic review. Arch Orthop Trauma Surg. 2017 Nov;137(11):1499-1513. 
9. Toon DH, Premchand RAX, Sim J, Vaikunthan R. Outcomes and financial implications of intraarticular distal radius fractures: a comparative study of open reduction internal fixation (ORIF) with volar locking plates versus nonoperative management. J Orthop Traumatol. 2017 Sep;18(3):229234.

10. Rundgren J, Bojan A, Mellstrand Navarro C, Enocson A. Epidemiology, classification, treatment and mortality of distal radius fractures in adults: an observational study of 23,394 fractures from the national Swedish fracture register. BMC Musculoskelet Disord. 2020 Feb 8;21(1):88.

11. Hevonkorpi TP, Launonen AP, Huttunen TT, Kannus P, Niemi S, Mattila VM. Incidence of distal radius fracture surgery in Finns aged 50 years or more between 1998 and 2016 - too many patients are yet operated on? BMC Musculoskelet Disord. 2018 Mar 2;19(1):70.

12. Jennifer L. Kelsey, PhD and Elizabeth J. Samelson. Variation in Risk Factors for Fractures at Different Sites. Curr Osteoporos Rep. 2009 December ; 7(4): 127-133

13. Ardouin L, Durand A, Gay A, Leroy M. Why do we use arthroscopy for distal radius fractures? Eur J Orthop Surg Traumatol. 2018 Dec;28(8):1505-1514.

14. Lameijer CM, Ten Duis HJ, Vroling D, Hartlief MT, El Moumni M, van der Sluis CK. Prevalence of posttraumatic arthritis following distal radius fractures in non-osteoporotic patients and the association with radiological measurements, clinician and patient-reported outcomes. Arch Orthop Trauma Surg. 2018 Dec;138(12):1699-1712.

15. Saving J, Enocson A, Ponzer S, Mellstrand Navarro C. External Fixation Versus Volar Locking Plate for Unstable Dorsally Displaced Distal Radius Fractures-A 3-Year Follow-Up of a Randomized Controlled Study. J Hand Surg Am. 2019 Jan;44(1):18-26.

16. Shimura H, Nimura A, Fujita K, Miyamoto T. Mid-Term Functional Outcome after Volar Locking Plate Fixation of Distal Radius Fractures in Elderly Patients. J Hand Surg Asian Pac Vol. 2018 Jun;23(2):238-242.

17. Fan J, Jiang B, Yuan F, Li SZ, Zhou JQ, Mei J, Cheng LM, Yu GR. Clinical effect of compound internal fixations in treating extreme distal radial fractures. Zhonghua Wai Ke Za Zhi. 2016 Oct 1;54(10):766771.

18. Karantana A, Scammell BE, Davis TR, Whynes DK. Cost-effectiveness of volar locking plate versus percutaneous fixation for distal radial fractures: Economic evaluation alongside a randomised clinical trial. Bone Joint J. 2015 Sep;97-B(9):1264-70.

19. Erhart S, Schmoelz W, Lutz M. Clinical and biomechanical investigation of an increased articular cavity depth after distal radius fractures: effect on range of motion, osteoarthrosis and loading patterns. Arch Orthop Trauma Surg. 2013 Sep;133(9):1249-55.

20. Vonier D, Enderle E, Sauerbier M. Treatment of distal radioulnar joint osteoarthritis. Unfallchirurg. 2012 Jul;115(7):589-97.

21. Lutz $M$, Krappinger $D$, Wambacher $M$, Rieger $M$, Pechlaner S.Arthritis predicting factors in distal intraarticular radius fractures. Arch Orthop Trauma Surg. 2011 Aug;131(8):1121-6. 
22. Matschke S, Wentzensen A, Ring D, Marent-Huber M, Audigé L, Jupiter JB. Comparison of angle stable plate fixation approaches for distal radius fractures. Injury. 2011 Apr;42(4):385-92.

23. Egol KA, Walsh M, Romo-Cardoso S, Dorsky S, Paksima N. Distal radial fractures in the elderly: operative compared with nonoperative treatment. J Bone Joint Surg Am. 2010 Aug 4;92(9):1851-7.

24. Kilic A, Ozkaya U, Kabukcuoglu Y, Sokucu S, Basilgan S. The results of non-surgical treatment for unstable distal radius fractures in elderly patients. Acta Orthop Traumatol Turc. 2009 MayJul;43(3):229-34.

25. Florek J, Kotela I, Georgiew F, Zieńczuk W, Rzeszutek T. Comparison of Radiographic Outcomes of Surgical Treatment in Patients with Distal Radial Fractures. Ortop Traumatol Rehabil. 2018 Dec 31;20(6):483-492.

26. Zyluk A, Janowski P, Szlosser Z, Puchalski P. Percutaneous K-wires vs palmar locking plate fixation for different types of distal radial fractures: a comparison of the outcomes of two methods to controll our guidelines. Handchir Mikrochir Plast Chir. 2018 Sep;50(5):319-325.

27. Zengin EC, Ozcan C, Aslan C, Bulut T, Sener M. Cast immobilization versus volar locking plate fixation of $A O$ type $C$ distal radial fractures in patients aged 60 years and older. Acta Orthop Traumatol Turc. 2019 Jan;53(1):15-18.

28. Yin SQ, Huang YP, Li MZ, Pan JD, Ding WQ, Wang X. Relationship between radiographic parameters and clinical outcomes of elderly patients with distal radius fractures. Zhongguo Gu Shang. 2018 Feb 25;31(2):141-144.

29. Knirk JL, Jupiter JB. Intra-articular fractures of the distal end of the radius in young adults. J Bone Jt Surg Am. 1986; 68(5):647-659.

30. Morasiewicz P, Dejnek M, Urbański W, Dragan St, Kulej M, Dragan SF. Radiological evaluation of ankle arthrodesis with Ilizarov fixation compared to internal fixation. Injury. 2017 Jul;48(7):16781683.

\section{Figures}




\section{Before}

\section{After}

\section{Figure 1}

Patient with volar plate fixation, AP view (a), and lateral view (b).
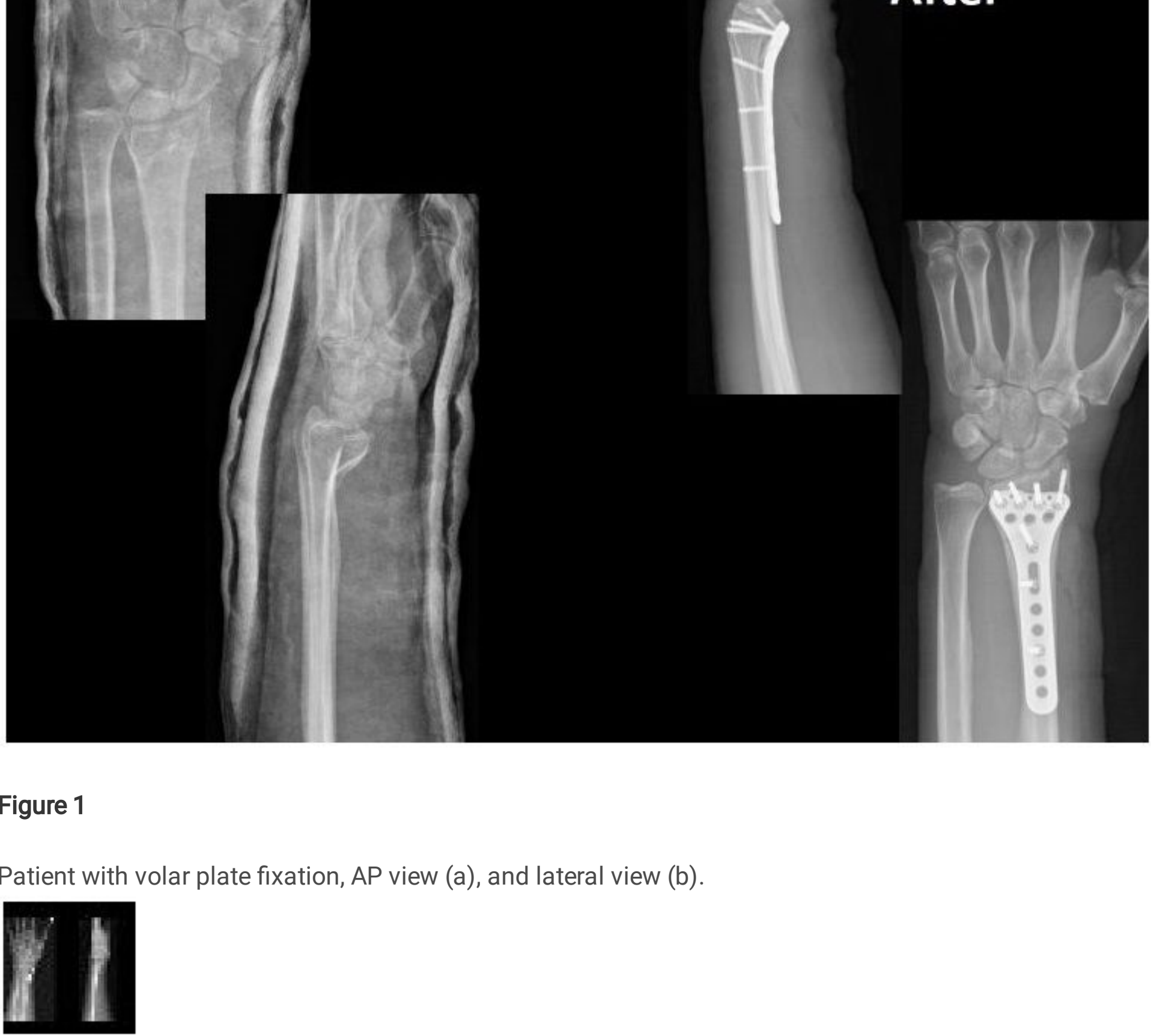

Figure 2

Patient with K-wires fixation, AP view (a), and lateral view (b). 


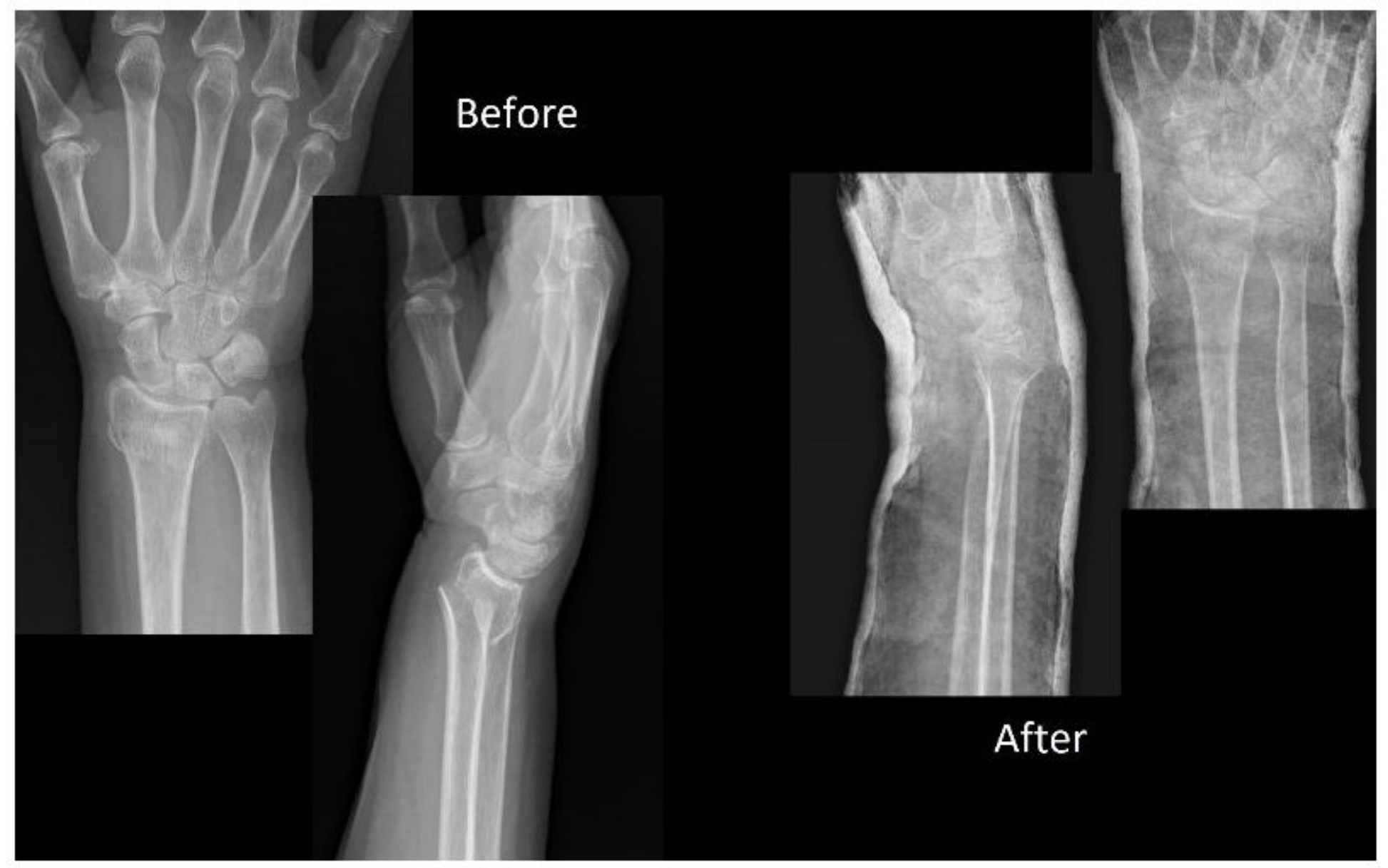

\section{Figure 3}

Patient with cast stabilization, AP view (a), and lateral view (b).

\section{Supplementary Files}

This is a list of supplementary files associated with this preprint. Click to download.

- data.xlsx 